\title{
The prevalence of hearing impairment within the Cape Town Metropolitan area
}

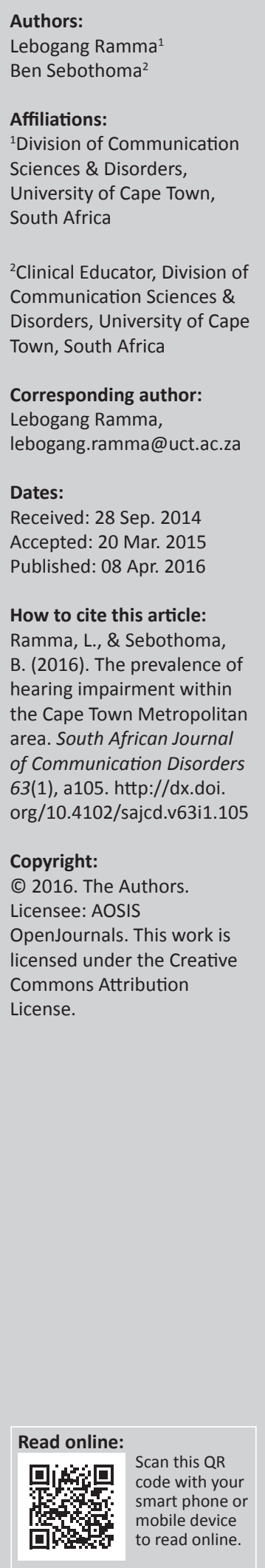

Background: There is a lack of data on the prevalence of hearing impairment in South Africa. Current data is unreliable as it is based on national census information which tends to underestimate the prevalence of hearing impairment.

Aim: The aim of this study was to estimate the prevalence of hearing impairment in the Cape Town Metropolitan area and to determine factors associated with hearing impairment.

Method: A cross-sectional household survey involving 2494 partcipants from 718 households was conducted between the months of February and October 2013. Random cluster sampling was used to select four health sub-districts from eight health sub-districts in the Cape Town Metropolitan area using a method of probability proportional to size (PPS). The survey was conducted according to the World Health Organization (WHO) Ear and Hearing Disorders Survey Protocol and the classifcation of hearing impairment matched the WHO's criteria for the grading of hearing impairment.

Results: The overall prevalence of hearing impairment in the population of this study was $12.35 \%$ (95\% CI: $11.06 \%-13.64 \%$ ) and prevalence of disabling hearing impairment was $4.57 \%$ (95\% CI: $3.75 \%-5.39 \%$ ) amongst individuals $\geq 4$ years old. The following factors were found to be associated with hearing impairment; male gender, age, hypertension, a history of head and neck trauma and a family history of hearing impairment.

Conclusion: Based on the data from communities surveyed during this study, hearing impairment is more prevalent than previously estimated based on national population census information. Interventions for the prevention of hearing impairment in these communities should focus on individuals with associated risk factors.

\section{Introduction}

Hearing is one of the essential senses for human communication. Hearing impairment at any stage of life can compromise the communication process and influence an individual's quality of life (Gondim et al., 2012). Hearing impairment in childhood can cause delays in the development of speech, language, and cognition which may later lead to educational disadvantage, social isolation and ultimately economic disadvantage (Finitzo, Albright \& O'Neal, 1998; Stevens et al., 2011; Yoshinaga-Itano, 2003). In adults, untreated hearing impairment has been linked to depression, anxiety and other psychological disorders (Kochkin \& Rogin, 2000), as well as an increased risk of dementia (Lin et al., 2011a).

Hearing impairment is a highly prevalent societal problem and it is one of the biggest contributors to the burden of disabilities in the world (Agrawal, Platz \& Niparko, 2008). Hearing impairment was ranked first in the category of health conditions associated with disability and among the leading causes of the global burden of disability in the World Disability Report of 2011 (World Health Organization [WHO], 2011). The WHO estimates that approximately 360 million persons live with disabling hearing impairment worldwide and that the majority of these people live in developing countries, specifically South Asia, Asia Pacific and Sub-Saharan Africa (WHO, 2012).

Various risk factors, also linked to specific stages of life, are associated with hearing impairment. In children, risk factors for hearing impairment include, but are not restricted to: low birth weight, craniofacial anomalies, neonatal infections such as cytomegalovirus, herpes hyperbilirubinemia requiring exchange transfusion, respiratory distress, prolonged mechanical ventilation, meningitis, a family history of hearing loss and low Apgar score (Saunders et al., 2007; Todd, 1994). For adults, the main risk factors for hearing impairment are advanced age and prolonged exposure to excessive noise (Isaacson \& Vora, 2003). Research also links some non-communicable diseases, e.g. cardiovascular disease and/or diabetes, and lifestyle factors such as smoking with increased risk of developing hearing impairment during adulthood (Fransen et al., 2008). Finally, exposure 
to ototoxic medications during the treatment of some health conditions such as cancer and drug-resistant tuberculosis have been shown to increase the risk of acquiring hearing impairment amongst both children and adults (Harris et al., 2011; Saunders et al., 2007; Whitehorn et al., 2014).

Population-based surveys that estimate the magnitude of hearing impairment are generally few, despite the fact that hearing impairment is considered to be a leading contributor to the burden of disability (Stevens et al.,2011). The lack of good estimates of the magnitude of hearing impairment makes it difficult to plan adequately for interventions and services aimed at individuals with hearing impairment. Currently, information on the prevalence of hearing impairment in South Africa is based primarily on data from the national population census (Statistics South Africa, 2003). Prevalence of hearing impairment estimates based on census data is typically based on self-report (or proxies' report) of hearing impairment by individuals interviewed, and this tends to underestimate the magnitude of the problem (Nondahl et al., 1998; Sindhusake et al., 2001). This study therefore aims to estimate the prevalence of hearing impairment in the Cape Town Metropolitan area using a survey protocol that includes actual assessment of hearing status.

\section{Method}

\section{Aim}

The aim of this study was to estimate the prevalence of hearing impairment in the Cape Town Metropolitan area as well as to investigate the factors associated with hearing impairment in the population of this study.

\section{Research design}

The study design made use of a cross-sectional population survey using the WHO's Ear and Hearing Disorders Survey Protocol (WHO, 1999). The survey was conducted between the months of February and October 2013 in the Cape Town Metropolitan area.

\section{Context}

The Cape Town Metropolitan area is one of the six health districts in the Western Cape. This health district is further divided into eight (health) sub-districts. The most recent national population census data of 2011 indicated that this metropolitan area has a population of 3740025 people evenly spread across these eight health sub-districts (Statistics South Africa, 2012).

\section{Participants}

Participants in this survey included all individuals in the selected households who gave consent or assent to take part in this study. Minors (children younger than 12 years old) were only included in the study if their parents or legal guardians gave consent. For older children ( $\geq 12$ years old) parental consent and assent from the child were obtained prior to being included in this study. Individuals were excluded from the study if they refused to give consent, were not at home after a second visit, were unable to understand either written or verbal instructions, or had cognitive impairment or communication difficulties.

\section{Sampling strategy}

Random cluster sampling was used to select four sub-districts (cluster) from the eight health sub-districts within the Cape Town Metropolitan area through a method of probability proportional to size (PPS). An aerial map of each of the health sub-districts to be surveyed was used to aid in selecting the households to be sampled. The distribution of types of residences for each sub-district was obtained from the national census database to determine the proportion of each residence type within the health sub-district (Statistics South Africa, 2012). The sample was further stratified according to the type of residence (i.e. free standing house, block of flats and shacks). In residences which contained several households (e.g. main house and a backyard shelter), only one of the households per residence was surveyed. In the case of a block of flats, one floor per block of flats was surveyed and each unit/flat was treated as a household. For each residence type strata, a total of eight streets were randomly selected. Fifteen residences were identified in each street using the map, starting from the first residence to the right of the street corner and then including every second residence from this point until 15 households were visited for each of the eight selected streets.

\section{Sample size}

The sample size for this study was determined using the WHO Ear and Hearing Disorders Survey Protocol (WHO, 1999). According to this protocol, assuming a prevalence of hearing impairment in the population of about $10 \%$, with a precision of $1.08 \%$ and a design effect factor of 2 , the required sample size for a simple random design (CI:95\%) should be 5924 persons (WHO, 1999). At least 1693 households were required to be visited to obtain the required sample size using an average household size of 3.50 persons in the Cape Town Metropolitan area (Statistics South Africa, 2012).

\section{Research personnel}

The research personnel comprised four teams of two senior Audiology students of the University of Cape Town per team, and two qualified audiologists with a total of 18 years of clinical experience between them. All of the students were senior Audiology students and therefore had adequate clinical skills required for the assessments required in this study. Furthermore, all the students were given additional training specific to the testing protocol used in this study to ensure consistency in assessment procedures and in the reporting of results.

\section{Ethical considerations}

This study adhered to the ethical principles as outlined in the Declaration of Helsinki (World Medical Association 
Declaration of Helsinki, 2008). Informed consent was requested from potential participants prior to participation in this study and they were informed that their participation was voluntary. All efforts were made to safeguard the confidentiality of participants' information and no specific identifying information was obtained for use in study reports. Participants were assured that they have the right to withdraw from this study at any time without negative consequences to themselves. Ethical approval to conduct the study was given by the Faculty Of Health Sciences Human Research Ethics Committee, University Of Cape Town (HREC REF 603/2012).

\section{Community entry}

Key stakeholders in the communities selected for this study were approached by the researchers after ethical approval to conduct the study was granted. This served to inform the stakeholders about the study and to request their support in encouraging community members to participate in this study. Key stakeholders included: Managers of health facilities, representatives of community forums as well as representatives of community health forums in the selected sub-districts. Community members were additionally informed about the study through posters placed at various points in the community and through flyers placed in mail boxes.

\section{Pilot study}

A pilot study was conducted in one of the four health subdistricts selected for this to test the study protocols. The outcome of the pilot study revealed that the assessment protocol proposed for this study was feasible in the communities being surveyed. Furthermore, the pilot study yielded information that optimised the efficient collection of data such as best test sequence when assessing participants' hearing status and the best days of the weeks and times of the day to conduct the survey.

\section{Procedures}

Research teams (in groups of two) approached selected households; explained the purpose of the study and procedures to be followed to the members of selected household with an invitation to participate. The research team entered the household and followed the following protocol, in households where the members (i.e. head of household or a guardian over the age of 18 years old) agreed and consented to be part of this study.

Obtain consent: The research team requested consent from each member of the household eligible to participate in this study. For young children $(<12$ years old), the parent or legal guardian was asked to give consent on behalf of the child to participate in the study. For older children ( $\geq 12$ years old, but $\leq 18$ years old), assent and consent was obtained from the child before being tested. Participants were asked to sign a consent form (or assent form where applicable) to indicate their willingness to participate in the study.
Assessment: Audiometric assessments proceeded in the following sequence: measurement of ambient noise levels in the room, obtaining pertinent background history information (refer to the Ear form, Appendix 1), audiometric assessment, feedback and referral to the nearest health facility (where applicable). Participants $\geq 4$ years were assessed using the following tests/procedures: otoscopic examination, tymapnometry and pure tone audiometry while partcipants aged 0-3 years were assessed using otoscopic examination, tymapnometry and Distortion Product Otoacoustic emissions (DPOAE) (refer to Appendix 2 for the detailed description of the assessment procedures).

\section{Classification of hearing loss}

Hearing impairment was classified according to the WHO grading of hearing impairment (refer to Table 1-A2, Appendix 2) criteria. Disabling hearing impairment was defined as a permanent unaided hearing threshold level in the better ear of $\geq 41 \mathrm{~dB}$ HL (for adults) and permanent unaided threshold level in the better ear of $\geq 31 \mathrm{~dB}$ or (children younger than 15 years old) (WHO, 2014). Hearing impairment was further classified according to the type (conductive, mixed or sensorineural hearing loss) for all participants $\geq 4$ years old.

\section{Reliability and validity}

To ensure consistency and reliability of data collected, all members of the research team were trained on the study protocol and procedures prior to commencement of the study. Furthermore, the data collected was verified by revisiting $10.7 \%$ of the selected households to confirm the following information: Head of the household (or the person who allowed the participants to enter the household), whether otoscopy, tympanometry and audiometry were done. The verification process showed good agreement (Kappa $=98 \%$ ) between data collected by different research teams and therefore the data was considered was reliable. Finally, all decisions regarding the diagnosis of hearing loss were made by a qualified audiologist and all decisions involving a diagnosis of hearing loss were forwarded to the second qualified audiologist for cross-checking. There was also good agreement between the two audiologists (kappa = 0.96). All of the equipment used in this study were calibrated prior to the start of this study and also underwent a daily biological calibration check prior to data collection sessions. Test procedures used in this study were routine audiological tests with established validity.

Data was captured on the hard copy version of the WHO/ PDH Ear and Hearing Disorders Examination Form (Version 7.1A), then transferred into an excel spread sheet by four research assistants. To ensure consistency when entering the data into an excel spread sheet, at least $10 \%$ of the data entered by each one of the research assistants was double checked by a different person to ensure accurate capturing of the data. 


\section{Data analysis}

Data analysis was performed using the STATA Data Analysis and Statistical Software package (Stata Corp LP, 2014) and both descriptive and inferential statistical methods were used. Tables and histograms were used to summarise and describe the results of the study. An independent $t$-test $(p=0.05)$ was used to compare hearing thresholds obtained under different ambient noise levels, whilst binomial logistic regression was used to model the relationship between hearing impairment and several explanatory variables; age, gender, level of education, a family history of hearing impairment, a history of stroke, a self-reported history of hypertension, a self-reported history of diabetes, smoking, a history of respiratory difficulties and a history of head and neck trauma. Binomial logistic regression was also done to estimate the probability (odds) of occurrence of hearing as a function of these explanatory variables.

\section{Results}

\section{Demographic profile}

A total of 791 households were approached and invited to take part in this study; 73 households either refused to participate (21 households) or no one was home on a second visit (52 households). It was not possible to find out the number of household members for 49 of the 73 households that were eligible but did not participate in this study. However, for the remaining 24 households in which household members were not assessed, the number of individuals per household ranged from 3 to 7 . The number of non-respondent households was distributed evenly across all the health districts surveyed (i.e. there was no district with a markedly high number of nonrespondent households).

Ultimately 718 households participated in this study and 2494 individuals ( $42 \%$ of the original study sample) from those households had their hearing screened; women comprised the majority of the participants $(60.9 \%)$ and individuals in the 10-19 years old age category constituted $22.9 \%$ of the sample, whilst individuals $60+$ years old constituted $7.6 \%$ of the sample (refer to Figure 1 for age and gender profile of participants in the study).

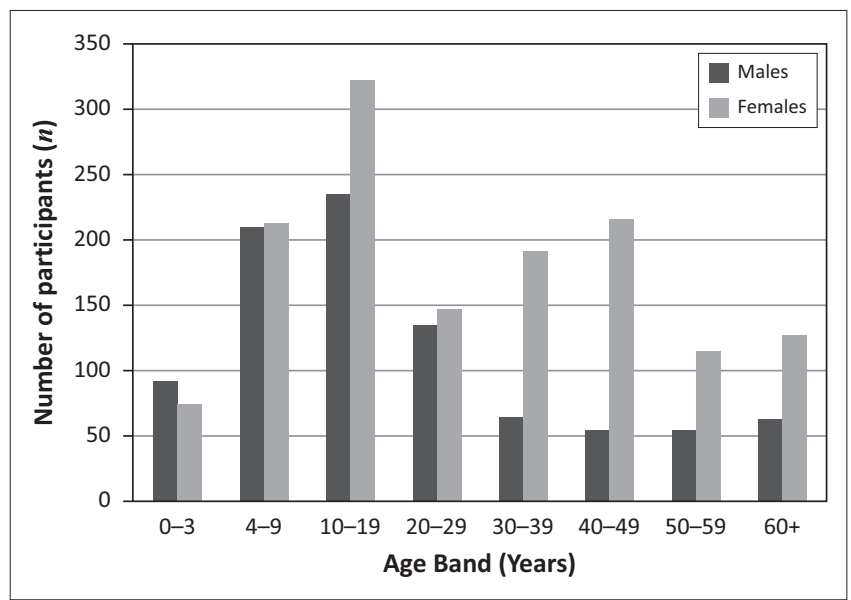

FIGURE 1: Age and gender profile of the participants $(n=2494)$.

\section{Ambient noise levels}

The average level of ambient noise during testing was $52.6 \pm 3.4 \mathrm{dBA}$. A $t$-test for independent samples $(p=0.05)$ showed no statistically significant relationship between the level of ambient noise during testing and the different categories of hearing impairment in this study $(p=0.163)$.

\section{Prevalence of hearing impairment}

Of the total number of participants to this study, $12.35 \%$ (95\% CI: $11.06 \%$ - 13.64\%) had some degree of hearing impairment. See Table 1 for a detailed presentation of hearing impairment according to different age categories.

\section{Disabling hearing impairment}

The prevalence of disabling hearing impairment amongst individuals who participated in this study was $4.57 \%$ (95\% CI: $3.75 \%-5.39 \%)$. As a group, males and participants older than 60 years old had a higher proportion of individuals with disabling hearing impairment than other groups of participants.

\section{Unilateral hearing loss}

The prevalence of unilateral hearing impairment in this study was $2.3 \%$ (95\% CI: $1.71 \%-2.89 \%)$. Tympanometry assessment also showed that participants in the 0-3 and 4-9 year old age categories had higher rates of abnormal tympanometry findings than other age categories. Refer to Table 3 for laterality of hearing impairment and tympanometry findings.

Association between hearing impairment and the following factors was investigated; age, gender, level of education, a family history of hearing impairment, prior episode of stroke (CVA), a self-reported history of hypertension, a self-reported history of diabetes, a prior history of respiratory difficulties, whether participant smokes or not, and a prior history of head and neck trauma. The results of this study showed that increased age, male gender, a family history of hearing impairment, a self-reported history of hypertension, and a prior history of head and neck trauma were associated with hearing impairment $(p<0.05)$ (refer to Table 4 below for more detailed information, including Odds Ration [OR]).

\section{Discussion}

The primary aim of this study was to estimate the prevalence of hearing impairment in selected health sub-districts within the Cape Town Metropolitan area. Using the WHO grading of hearing impairment classification (WHO, 2014), it was estimated that overall prevalence of hearing impairment in this study was $12.35 \%$ (95\% CI: $11.06 \%$ - 13.64\%). Age, gender, a family history of hearing impairment, a selfreported history of hypertension, and a history of prior head and neck trauma were main factors associated with hearing impairment. 
TABLE 1: Percentage (\%) of participants by degree of hearing impairment and age category.

\begin{tabular}{|c|c|c|c|c|c|c|}
\hline \multirow{2}{*}{$\begin{array}{l}\text { Disabling hearing impairment: } \\
\text { Age band (Years) }\end{array}$} & \multirow[t]{2}{*}{$n$} & \multirow{2}{*}{$\begin{array}{c}\text { No impairment } \\
0-25 \mathrm{~dB}(\%)\end{array}$} & \multirow{2}{*}{$\begin{array}{c}\text { Slight impairment } \\
26-30 \text { or } 40 \mathrm{~dB}(\%)\end{array}$} & \multirow{2}{*}{$\begin{array}{c}\text { Moderate } \\
31 \text { or } 41-60 \mathrm{~dB}(\%)\end{array}$} & \multirow{2}{*}{$\begin{array}{c}\text { Severe } \\
61-80 \mathrm{~dB}(\%)\end{array}$} & \multirow{2}{*}{$\begin{array}{c}\text { Profound } \\
\geq 81 \mathrm{~dB}(\%)\end{array}$} \\
\hline & & & & & & \\
\hline $0-3^{*}$ & 174 & 88.5 & 11.4 & $\mathrm{~N} / \mathrm{A}$ & N/A & N/A \\
\hline $4-9$ & 430 & 95.8 & 3.3 & 0.5 & 0.5 & 0.0 \\
\hline $10-19$ & 570 & 97.5 & 1.1 & 1.1 & 0.0 & 0.4 \\
\hline $20-29$ & 430 & 92.1 & 6.0 & 0.9 & 0.9 & 0.0 \\
\hline 30-39 & 260 & 86.9 & 9.2 & 3.1 & 0.8 & 0.0 \\
\hline $40-49$ & 270 & 78.5 & 11.1 & 7.4 & 2.2 & 0.7 \\
\hline $50--59$ & 170 & 67.1 & 22.4 & 7.1 & 2.4 & 1.2 \\
\hline $60+$ & 190 & 61.1 & 21.1 & 12.6 & 4.2 & 1.1 \\
\hline Total/Overall & 2494 & 87.7 & 7.8 & 3.1 & 1.1 & 0.3 \\
\hline
\end{tabular}

* It was not possible to differentiate between the degrees of hearing impairment in children 0-3 years due to the assessment procedures used. Therefore $11.4 \%$ reflects the proportion of participants who obtained "refer" as described above (and not necessarily a slight hearing impairment).

TABLE 2: Proportion of persons with disabling hearing (\%) loss by gender and age categories.

\begin{tabular}{lccc}
\hline Age Band (Years) & Male (\%) & Female (\%) & Overall (\%) \\
\hline $4-9$ & 1.9 & 0.0 & 0.9 \\
$10-19$ & 0.0 & 0.6 & 1.1 \\
$20-29$ & 1.5 & 1.4 & 1.9 \\
$30-39$ & 9.4 & 2.1 & 3.8 \\
$40-49$ & 3.7 & 6.5 & 10.4 \\
$50-59$ & 18.5 & 10.5 & 10.6 \\
$60+$ & 29.0 & 11.1 & 17.9 \\
Overall & $\mathbf{5 . 1}$ & $\mathbf{3 . 1}$ & 4.6 \\
\hline
\end{tabular}

TABLE 3: Laterality of hearing loss \& Tympanometry.

\begin{tabular}{lccc}
\hline Age Band (Years) & Unilateral HI (\%) & Bilateral HI (\%) & $\begin{array}{c}\text { Bilateral Type B* } \\
\text { Tympanogram (\%) }\end{array}$ \\
\hline $0-3$ & 2.4 & 11.5 & 16.1 \\
$4-9$ & 1.4 & 4.2 & 5.6 \\
$10-19$ & 0.4 & 2.5 & 1.8 \\
$20-29$ & 2.9 & 7.9 & 2.3 \\
$30-39$ & 3.1 & 13.1 & 0.8 \\
$40-49$ & 3.7 & 21.5 & 4.4 \\
$50-59$ & 1.2 & 32.9 & 4.7 \\
$60+$ & 6.4 & 38.9 & 2.1 \\
\hline Overall & $\mathbf{2 . 3}$ & $\mathbf{1 2 . 3}$ & 3.4 \\
\hline
\end{tabular}

*, A 'type $\mathrm{B}^{\prime}$ tympanogram curve is indicative of a non-compressible fluid within the middle ear space (otitis media), tympanic membrane perforation, or debris within the external ea canal (cerumen).

Source: Mikolai, T.K., Duffey, J., \& Adlin, D. (2006). A guide to tympanometry for hearing screening: Maico Diagnostic. Retrieved January 23, 2014 from http://www.maico-diagnostic. com/eprise/main/_downloads/com_en/Documentation/Guide.Tymp.pdf

TABLE 4: Factors associated with hearing loss.

\begin{tabular}{lcc}
\hline Hearing loss & Odds ratio $(95 \% \mathrm{Cl})$ & $p$-value \\
\hline Age & $1.03(1.02,1.04)$ & $<0.001^{*}$ \\
Gender & $1.62(1.08-2.41$ & $0.032^{*}$ \\
Education & $0.82(0.52,1.30)$ & 0.404 \\
Family History & $3.02(1.93,4.73)$ & $<0.001^{*}$ \\
CVA stroke & $0.49(0.19,1.24)$ & 0.131 \\
Hypertension & $2.05(1.15,3.65)$ & $0.015^{*}$ \\
Diabetes & $0.56(0.26,1.20)$ & 0.139 \\
Smoker & $1.30(0.81,2.08)$ & 0.273 \\
Respiratory difficulties & $1.36(0.76,2.41)$ & 0.296 \\
Head \& Neck Trauma & $1.94(1.00,3.78)$ & $0.05^{*}$ \\
\hline
\end{tabular}

$*$, statistically significant $(p=0.05)$.

While the estimates of the prevalence of hearing impairment in this study were consistent with that of Stevens et al., (2011), it was noted that it was slightly lower than prevalence rates reported in previous studies which used the WHO Ear and Hearing Disorders Survey protocol (WHO, 1999).
For instance, a study conducted in Brazil (Béria et al., 2007) reported prevalence rates of $26.1 \%$ (overall hearing impairment) and 6.8\% (disabling hearing impairment). An Egyptian study by Abdel-Hamid, Khatib, Aly, Morad, and Kamel (2007) reported prevalence rates of $16.0 \%$ (overall hearing impairment) and $2.9 \%$ for disabling hearing impairment, whilst a study by Westerberg et al. (2008) done in Uganda also reported much higher prevalence rates for disabling hearing impairment than reported in the current study: $11.7 \%$ in adults and $10.2 \%$ in children respectively.

It is generally difficult to compare prevalence estimates across different studies even when using similar protocols and definitions of hearing impairment. The difficulty in such comparisons stems from differences in demographic characteristics of participant cohorts and study contexts (Lin, Thorpe, Gordon-Salant and Ferrucci (2011b). Therefore, it was expected that prevalence estimates in this study would be somewhat different from those of other studies. However, part of the reasons for differences in the prevalence rates reported in previous studies and the current study may also have to do with the criteria used to ascertain the degree of hearing impairment in children younger than four years old. It was not clear how some of these studies confirmed the degree and type of hearing impairment in children. For instance, the Brazilian study only used pure tone audiometry (and did not use tympanometry) and therefore would have had difficulties in confirming hearing impairment in participants in this age category. The Egyptian study (Abdel-Hamid et al., 2007) on the other hand, deviated from the recommended WHO Ear and Hearing Disorders protocol and used only otoacoustic emission and tympanometry to assess hearing impairment, which makes it extremely difficult to ascertain degree and type of hearing impairment across all age categories.

Consistent with the findings of previous studies (Lin et al., 2011b; McMahon, Kifley, Rochtchina, Newall \& Mitchell, 2008; Saunders et al., 2007), this study found that increasing age was associated with hearing impairment. In one American study, Lin et al., (2011b) reported that about two thirds of adults aged 70 years and older had some degree of hearing impairment. Regarding association between gender and hearing impairment, previous research studies (Caban, Lee, Gómez-Marín, Lam \& Zheng, 2005; Smeeth et al., 2002) have reported a higher prevalence of hearing impairment in males than females, and the same trend was also observed in 
this study. It is worth noting though that gender differences in the prevalence of hearing impairment has been reported even in populations with no evidence of noise-induced hearing loss (Pearson et al., 1995) therefore this difference cannot simply be attributed the fact that men generally tend to be exposed to noisy activities.

A family history of hearing impairment and a history of head and neck trauma were other factors that were found to be associated with hearing impairment in this study, which is also consistent with the findings of previous studies (Fitzgerald, 1996; McMahon et al., 2008; Ottaviano et al., 2009). In an Australian study involving 2669 individuals aged 50 years and older, McMahon et al., (2008) reported that a family history of hearing impairment (especially a maternal family history of hearing impairment) was strongly associated with moderate to severe age-related hearing impairment. With respect to a history of head and neck trauma, hearing loss following trauma to the head and neck areas has been documented in literature (Fitzgerald, 1996; Ottaviano et al., 2009).

Results indicate that participants who reported a history of hypertension, in this study, were twice as likely to have hearing impairment as participants who did not report hypertension. This finding was consistent with those of previous studies (Chang et al., 2011; Chao, 2004; Fransen et al., 2008; Kakarlapudi, Sawyer \& Staecker 2003). An unexpected finding in this study was the lack of statistically significant association between a history of diabetes and hearing impairment. This is in spite of the fact that there are now several studies that have reported a plausible association between diabetes and hearing impairment (Jáuregui-Renaud, Sánchez, Ibarra Olmos \& González-Barcena, 2009; Pemmaiah \& Srinivas, 2011; Thimmasettaiah \& Shankar, 2012) Possible reasons for the apparent lack of association between diabetes and hearing impairment may have to do with the small number of participants who reported a history of diabetes in this study. Some of the participants may not have been aware that they are diabetic or may have simply chosen not to disclose their diabetes status.

The findings of this study must be interpreted with caution given its methodological limitations. These included unfavourable testing environments as audiological assessment done outside of audiometric booths is bound to be challenging. Specific to this study, the fact that the test environment varied from one household to the next, introduced a further challenge to this study. However, the study protocol was very stringent regarding ambient noise levels that were considered acceptable for a hearing assessment to be conducted. For instance, if the environment was found to be too noisy (i.e. ambient noise $>55 \mathrm{~dB}$ ) then a hearing assessment was not done at all or was terminated. Furthermore, an analysis of the results also revealed that there was no association between a participant being diagnosed with hearing impairment or having a particular degree of hearing impairment and the level of noise in the room.

Another limitation of this study was the small sample size which compromised the representativeness of the study sample. The calculated sample for this study was 5924 participants. However, only 42\% (2494 participants) of this sample was achieved during this study. Data collection for this study had to be terminated prematurely before achieving the required sample size due to a flare-up of gang-related violence in the areas selected for this survey. This may have led to the resultant study sample being biased in favour of female participants and participants under the age of 20 years old, and this may have potentially introduced a bias that could have led to an underestimation of the prevalence of hearing impairment in this study. However, despite a possibly biased sample, the trends revealed by the data in this study were consistent with those reported in previous studies, i.e. more hearing impairment in males than females, as well as an association between hearing impairment and advanced age.

\section{Conclusions}

This study showed that the prevalence of hearing impairment in the communities surveyed is much higher than current estimates obtained through national census. Main factors associated with hearing impairment were also identified. Therefore, in spite of its limitations, this study represents one of the few population-based surveys of hearing impairment done in South Africa. The findings of this study could therefore be useful in planning for prevention and interventions services for hearing impairment, especially in the Cape Town Metropolitan area. It is also hoped that the findings of this study will stimulate interest among the South African research community to conduct similar research studies in other parts of the country (especially in rural areas) to obtain a more complete picture of the magnitude of hearing impairment on a national scale. This will yield information that could be used to lobby for resources that are required for prevention and intervention services for hearing impairment.

\section{Acknowledgements}

The authors would like to acknowledge and thank all the individuals who took time to participate in this study. Also a special thanks to the following individuals who dedicated their time and energy to ensure that this study became a reality: Sithembile Ndlela, Vuyisani Sholo, Fatima Ally, Rhofiwa Netsifhire, Sivuyile Jange \& Siphesihle Mcetywa, Muneebah Benjamin, Maserame Masemola, Jareth Andreas, Bongile Langa, Kerry-Ann Youngman, Zenzo Chakara, Chárnae Pieterse and Jane Le Roux.

\section{Competing interests}

The authors declare that they have no financial or personal relationships which may have inappropriately influenced them in writing this article.

\section{Authors' contributions}

L.R. (University of Cape Town) was the project leader. He conceptualised the project, participated in the collection 
of data for the study and wrote the initial draft of the manuscript. B.S. (University of Cape Town) was the data collection manager. He coordinated data collection activities and reviewed and gave input in drafts of this manuscript.

\section{References}

Abdel-Hamid, O., Khatib, O.M.N., Aly, A., Morad, M., \& Kamel, S. (2007). Prevalence and patterns of hearing impairment in Egypt: A national household survey. Eastern Mediterranean Health Journal, 13(5), 1170-1180.

Agrawal, Y., Platz, F.A., \& Niparko, J.K. (2008). Prevalence of hearing loss and differences by demographic characteristics among US adults: Data from the National Health and Nutrition Examination Survey, 1999-2004. Archives of Internal Medicine, 168(14), 1522-1530. http://dx.doi.org/10.1001/archinte.168.14.1522

Béria, J.U., Raymann, B.C.W., Gigante, L.P., Figueredo, A.C.L., Jotz, G., Roithman, R. et al. (2007). Hearing impairment and socioeconomic factors: A population-based survey of an urban locality in Southern Brazil. Pan American Journal of Public Health, 21(6), 381-387. http://dx.doi.org/10.1590/S1020-49892007000500006

Caban, A.J., Lee, D.J., Gómez-Marín, O., Lam, B.L., \& Zheng, D.D. (2005). Prevalence of concurrent hearing and visual impairment in US adults: The National Health Interview Survey, 1997-2002. American Journal of Public Health, 95, 1940-1942. http://dx.doi.org/10.2105/AJPH.2004.056671

Chang, T., Liu, C., Huang, K., Chen, R., Lai, J., \& Bao, B. (2011). High-frequency hearing loss occupational noise exposure and hypertension: A cross-sectional study in male workers. Environmental Health, 10, 1-9. http://dx.doi.org/10.1186/1476069x-10-35

Chao, T. (2004). Case report: Sudden sensorineural hearing loss after rapid reduction of blood pressure in malignant hypertension. Annals of Otology, Rhinology \& Laryngology, 113, 73-75. http://dx.doi.org/10.1177/000348940411300116

Finitzo, T, Albright, K., \& O'Neal, J. (1998). The newborn with hearing loss: Detection in the nursery. Pediatrics, 102(6), 1452-1460. http://dx.doi.org/10.1542/ peds.102.6.1452

Fitzgerald, D.C. (1996). Head trauma: Hearing loss and dizziness. The Journal of Trauma: Injury, Infection, and Critical Care, 40(3), 488-496. http://dx.doi. org/10.1097/00005373-196603000-00034

Fransen, E., Topsakal, V., Hendrickx, J.J., Van Laer, L., Huyghe, J.R., \& Van Eyken, E. (2008). Occupational noise, smoking, and a high body mass index are risk factors for age-related hearing impairment and moderate alcohol consumption is protective: A European population-based multi-center study. Journal of the Association for Research in Otolaryngology: JARO.

Gondim, L.M.A., Balen, S.A., Zimmermann, K.J., Pagnossin, D.F., Fialho I de, M., \& Roggia, S.M. (2012). Study of the prevalence of impaired hearing and its determinants in the city of Itajaí, Santa Catarina State, Brazil. Brazilian Journal of Otorhinolaryngology, 78(2), 27-34. http://dx.doi.org/10.1590/S1808 86942012000200006

Harris, T., Bardien, S., Schaaf, H.S., Petersen, L., De Jong, G. \& Fagan, J.J. (2011). Aminoglycoside-induced hearing loss in HIV-positive and HIV-negative multidrugresistant tuberculosis patients. South African Medical Journal, 102(6), 363-366.

Isaacson, T.E., \& Vora, N.M. (2003). Differential diagnosis and treatment of hearing loss. American Academy of Family Physicians, 68(6), 1125-1132.

Jáuregui-Renaud, K., Sánchez, B., Ibarra Olmos, A., \& González-Barcena, D. (2009). Neurootologic symptoms in patients with type 2 diabetes mellitus. Diabetes Research and Clinical Practice, 84, 45-47. http://dx.doi.org/10.1016/j.diabres.2009.02.015

Kakarlapudi, V., Sawyer, R., \& Staecker, H. (2003). The effect of diabetes on sensorineural hearing loss. Otology and Neurology, 24, 382-386. http://dx.doi. org/10.1097/00129492-200305000-00006

Kochkin, S., \& Rogin, C. (2000). Quantifying the obvious: The impact of hearing aids on quality of life. Hearing Review, 7(1), 8-34

Lin, F. R., Metter, E. J., O'Brien, R. J., Resnick, S. M., Zonderman, A. B., \& Ferrucci, L. (2011a). Hearing loss and incident dementia. Archives of Neurology, 68, 214-220. http://dx.doi.org/10.1001/archneurol.2010.362

Lin, F.R., Thorpe, R., Gordon-Salant, S., \& Ferrucci, L. (2011b). Hearing loss prevalence and risk factors among older adults in the United States. The Journals of Gerontology. Series a, Biological Sciences and Medical Sciences, 66(5), 582-590. $\mathrm{http}: / / \mathrm{dx}$.doi.org/10.1093/gerona/glr002

Maclennan-Smith, F.J., Swanepoel de, W., \& Hall, J.W. 3rd. (2013). Validity of diagnostic pure tone audiometry without a sound-treated environment in older adults, International Journal of Audiology. 52(2), 66-73. http://dx.doi.org/10.310 9/14992027.2012.736692

McMahon, C.M., Kifley, A., Rochtchina, E., Newall, P., \& Mitchell, P. (2008). The contribution of family history to hearing loss in an older population. Ear and Hearing, 29(4), 578-584. http://dx.doi.org/10.1097/AUD.0b013e31817349d6
Mikolai, T.K., Duffey, J., \& Adlin, D. (2006). A guide to tympanometry for hearing screening: Maico Diagnostic. Retrieved January 23, 2014, from http:// www.maico-diagnostic.com/eprise/main/downloads/com en/Documentation/ Www.maico-diag

Nondahl, D.M., Cruickshanks, K.J., Wiley, T.L., Tweed, T.S., Klein, R., \& Klein, B.E. (1998). Accuracy of self-reported hearing loss, Audiology, 37(5), 295-301.http:// dx.doi.org/10.3109/00206099809072983

Ottaviano, G., Marioni, G., Marchese-Ragona, R., Trevisan, C.P., De Filippis, C., \& Staffieri, A. (2009). Anosmia associated with hearing loss and benign positional vertigo after head trauma. Acta Otorhinolaryngologica Italica, 29, 270-273.

Pearson, J.D., Morrell, C.H., Gordon-Salant, S., Brant, L.J., Metter, E.J., Klein, L.L., et al. (1995). Gender differences in longitudinal study of age-associated hearing loss, The Journal of the Acoustical Society of America, 97(2), 1196-205.

Pemmaiah, K., \& Srinivas, D. (2011). Hearing loss in Diabetes Mellitus. Internationa Journal of Collaborative Research on Internal Medicine and Public Health, 3, 725-732. Retrieved April 08, 2014 from http://iomcworld.com/ijcrimph/ijcrimphv03-n10-03.htm

Saunders, J.F., Vaz, S., Greinwald, J.H., Lai, J., Mortin, L., \& Mojica, K. (2007). Prevalence and etiology of hearing loss in rural Nicaraguan children, Laryngoscope, 117(3), 387-398. http://dx.doi.org/10.1097/MLG.0b013e31802e3726

Sindhusake, D., Mitchell, P., Smith, W., Golding, M., Dewal, P., Hartley, D. et al. (2001). Validation of self-reported hearing loss: The Blue Mountains Hearing Study. International Journal of Epidemiology, 30(6), 1371-1378. http://dx.doi. org/10.1093/ije/30.6.1371

Smeeth, L., Fletcher, A.E., Ng, E.S., Stirling, S., Nunes, M., Breeze, E., et al. (2002). A reduced hearing, ownership, and use of hearing aids in elderly people in the UK - the MRC Trial of the assessment and management of older people in the community: A cross-sectional survey, Lancet, 359, 1466-1470. http://dx.doi. org/10.1016/S0140-6736(02)08433-7

STATA CORP LP. (2014). STATA: Data analysis and statistical software package. College Station.

Statistics South Africa. (2003). Census 2001: Census in brief. Pretoria.

Statistics South Africa. (2012). Census 2011 release. Pretoria.

Stevens, G., Flaxman, S., Brunskill, E., Mascarenhas, M., Mathers, C.D., \& Finucane, M. (2011). Global and regional hearing impairment prevalence: An analysis of 42 studies in 29 countries. The European Journal of Public Health.

Swanepoel de, W., Koekemoer, D., \& Clark, J. (2010a). Intercontinental hearing assessment - A study in tele-audiology, Journal of Telemedicine and Telecare, 16(5), 248-252. http://dx.doi.org/10.1258/jtt.2010.090906

Swanepoel de, W., Mngemane, S., Molemong, S., Mkhwanazi, H., \& Tushini, S. (2010b). Hearing Assessment - Reliability, accuracy, and efficiency of automated audiometry. Telemedicine \& e-Health, 16(5).

Thimmasettaiah, N.B., \& Shankar, R. (2012). A one year prospective study of hearing loss in diabetes in general population. Translational Biomedicine, 3, 1-8.

Todd, N.W. (1994). At-risk populations for hearing impairment in infants and young children. International Journal of Pediatric Otorhinolaryngology, 29, 11-21. http://dx.doi.org/10.1016/0165-5876(94)90104-X

Westerberg, B.D., Lee, P.K., Lukwago, L., Zaramba, S., Bubikere, S., \& Stewart, I. (2008). Cross-sectional survey of hearing impairment and ear disease in Uganda. Journa of Otolaryngology - Head \& Neck Surgery, 37(6), 753-758

Whitehorn, H., Sibanda, M, Lacerda, M, Spraken, T., Ramma, L., Dalvie, S., et al. (2014). High prevalence of cisplatin-induced ototoxicity in Cape Town, South Africa. South African Medical Journal, 104(4), 288-291. http://dx.doi.org/10.7196/samj.7389

World Health Organization (WHO). (1999). WHO ear and hearing disorders survey protocol for a population-based survey of prevalence and causes of deafness and hearing impairment and other causes. Retrieved October 12, 2012, from http// www.who.int/pbd/pdh/pdh_home.htm

World Health Organization. (2011). World Report on Disability. Retrieved October 8 2012, from http://www.who.int/disabilities/world_report/2011/report/en/

World Health Organization. (2012). WHO global estimates on prevalence of hearing loss. Retrieved October 10, 2012, from http://www.who.int/pbd/deafness/WHO_ GE_HL.pdf

World Health Organization. (2014). Grades of hearing impairment. Retrieved October 10, 2014, from http://www.who.int/pbd/deafness/hearing_impairment grades/ en/index.html

World Medical Association Declaration of Helsinki. (2008). Ethical principles for medical research involving human subjects. Retrieved October 12, 2012, from http://www.wma.net/en/30publications/10policies/b3/index.html

Yoshinaga-Itano, C. (2003). From screening to early identification and intervention: Discovering predictors to successful outcomes for children with significant hearing loss, Journal of Deaf Studies and Deaf Education, 8(1), 11-30. http://dx.doi. org/10.1093/deafed/8.1.11 


\section{Appendix 1}

\section{WHO/PDH Ear and Hearing Disorders Examination form}

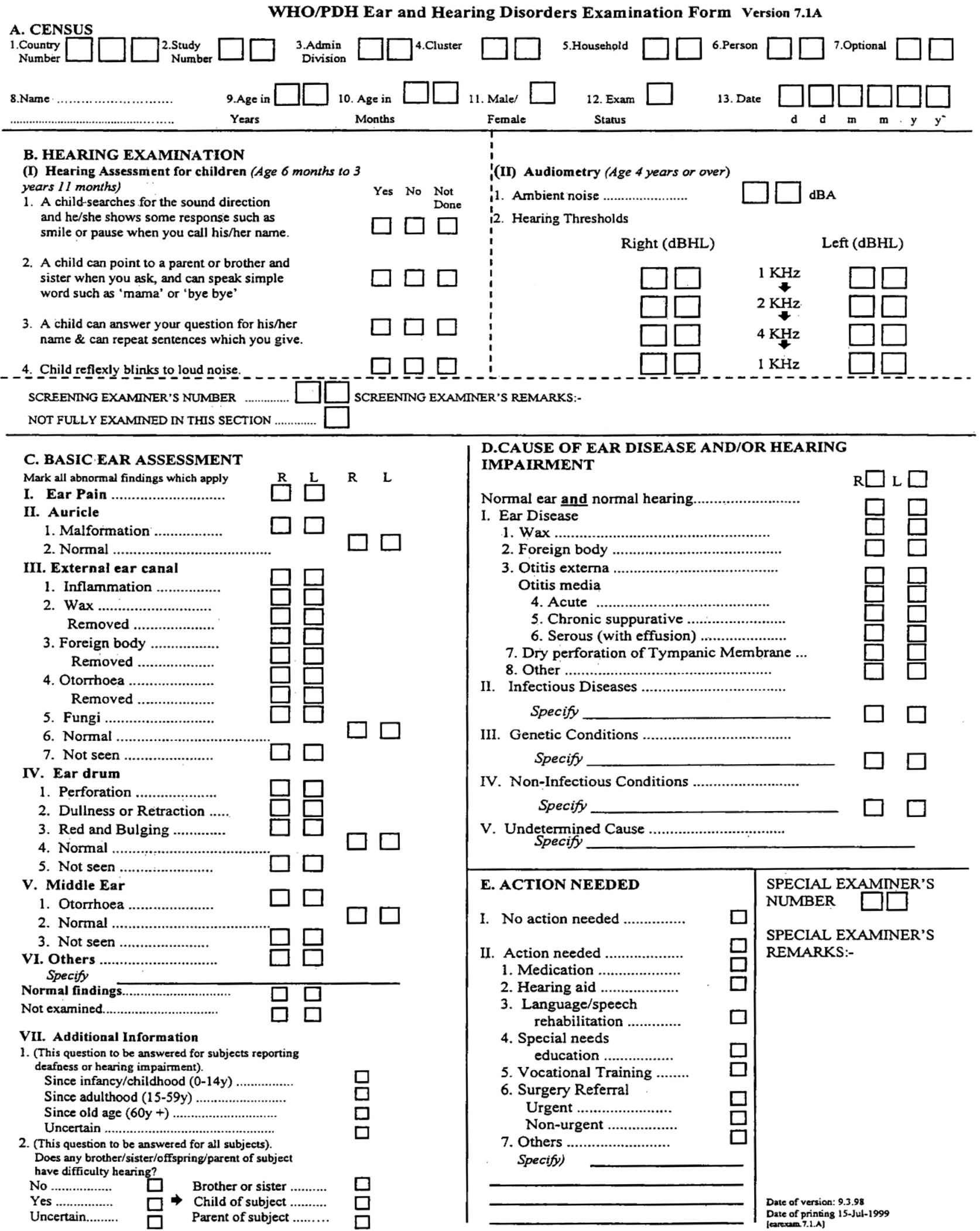

Source: World Health Organization (WHO). (1999). WHO ear and hearing disorders survey protocol for a population-based survey of prevalence and causes of deafness and hearing impairment and other causes. Retrieved October 12, 2012, from http//www.who.int/pbd/pdh/pdh_home.htm

Note: Section B of this form was not used. 


\section{Appendix 2}

\section{Instrumentation \& Assessment Procedures}

\section{Instrumentation}

- Otoscope Heinne S700 mini

- GSI 39 Tympanometer Screener

- GSI 17 Screening Audiometer

- GSI AUDIOscreener+

- KUDUwave 5000 Audiometer

- Bruel \& Kjær 2239 Type II Integrating Sound Level meter (Denmark)

- WHO/PDH Ear and Hearing Disorders Examination Form (Version 7.1A)

\section{Assessment procedures}

Measurement of noise levels

Measurement of ambient noise level in the room was measured using Bruel \& Kjær 2239 Type II Integrating Sound Level meter (Bruel \& Kjær, Denmark) prior to the start of assessment. If noise levels were deemed suitable for pure tone hearing screening $(\leq 50 \mathrm{dBA} \pm 5 \mathrm{dBA})$ then the researcher proceeded to interview participants to get background information about their hearing/auditory status (i.e. case history information) followed by audiometric assessment.

\section{Audiometric assessment}

All audiometric equipment used in this study were calibrated prior to the commencement of this study and were used exclusively in this study for the duration of the survey. Furthermore, biological calibration of each piece of equipment was performed every morning before the equipment was used. Audiometric assessment were conducted by trained research assitants (all senior audiology students) under close supervision of a qualified audiologist. Audiometric testing was only done when the ambient noise in the room was deemed suitable for a hearing assessment. The following assessment procedures were followed:

Individials $\geq 4$ years old; otoscopic examination was conducted using Heinne S700 mini otoscope (Heinne Optotechnic, Germany) to view and assess the external ear canal and tympanic membrane status. Otoscopic examination was followed by tympanometry screening using GrasonStadler (GSI 39 screening tympanometer (GSI, Minnesota, United States of America) to check mobility of the tympanic membrane and assess middle ear statusand pure tone audiometry screening. Hearing status was screened using GSI 17 portable audiometer (GSI, Minnesota, United States of America) with standard TDH 39 headphones fittted with Amplivox Audiocups (Amplivox Limited, United Kindom). A screening level of $30 \mathrm{~dB}$ HL (with a $60 \mathrm{~dB} \mathrm{HL} 1 \mathrm{kHz}$ as the first tone presented) at the following frequencies; $0.5,1$, $2 \& 4 \mathrm{kHz}$ was used and audiometric test started on the ear that the participant considered their best ear. Participants were asked to raise their hands everytime they hear the test tone. Partcipant was considerd to have "passed" the hearing screening (i.e. no hearing impairment) if they responded to at least 3 out of the 4 test frequencies in each ear and a "refer" result (i.e. hearing impairment suspected) was when the participants failed to repond to at least 2 of the test frequencies in at least one ear.

Individuals who obtained "refer" results underwant further assessment (diagnostic) using a KUDUwave 5000 audiometer (GeoAxon, Pretoria, South Africa) to confirm the degree and type of hearing impairment. This audiometer was selected because it is capable of live monitoring of environmental noise and it also has enhanced noise attenuation capability through the use of a combination of insert earphone and circumaural earphone which has implications for accuracy of test results (Swanepoel, Mngemane, Molemong, Mkhwanazi \& Tushini, 2010b). Furthermore, it has been shown to yield test results that a comparable to those that are obtained in a standard sound treated environment (Maclennan-Smith, Swanepoel de \& Hall, 2013; Swanepoel, Koekermoer \& Clark, 2010a).

For children $\leq 3$ years old; the following assessment procedures were followed; otoscopic examination (Heinne S700 mini otoscope), tympanometry screening (GSI 39 screening tympanometer [GSI, Minnesota, United States of America]). Distortion Product Otoacoustic Emissions (DPOAE) screening using GSI AUDIOscreener+ (GSI, Minnesota, United States of America) was used instead of pure tone audiometry. A child was considered to have "passed" the hearing screening if a DPOAE response was obtained to at least 2 of the 3 screening frequencies $(1,2 \& 3 \mathrm{kHz})$ plus a Type A tympnogram in both ears. A "refer" results was no response to at least 2 of the screening frequencies with or without an abnormal tympanometry results in at least one ear.

All groups of partcipants (i.e $\leq 3$ years old or $\geq 4$ years old), with abnormal audiometric test results following the hearing screening and or diagnostic assessment were referred to the nearest primary health care facility to initiate the process of getting appropriate services for their hearing impairment. All assessment information was recorded on the relevant sections of the $\mathrm{WHO} / \mathrm{PDH}$ Ear and Hearing Disorders Examination Form (Version 7.1A) (WHO, 1999).

\section{Classification of hearing loss}

Disabling hearing impairment in this study was defined as a permanent unaided threshold level in the better ear of $\geq 41$ dB HL (for adults) and permanent unaided threshold level in the better ear of $\geq 31 \mathrm{~dB}$ or (children younger than 15 years old) (refer to Table 1B below). 
Table 1-A2: WHO Criteria for grading of hearing loss.

\begin{tabular}{|c|c|c|c|}
\hline Grade of Impairment & $\begin{array}{l}\text { Corresponding } \\
\text { audiometric ISO value }\end{array}$ & Performance & Recommendations \\
\hline 0 - No impairment & $25 \mathrm{~dB}$ or better (better ear) & No or very slight hearing problems & \\
\hline 1 - Slight impairment & 26-40 dB (better ear) & $\begin{array}{l}\text { Able to hear and repeat words spoken in } \\
\text { normal voice at } 1 \text { meter }\end{array}$ & Counselling. Hearing aids may be needed \\
\hline 2 - Moderate impairment & 41-60 dB (better ear) & $\begin{array}{l}\text { Able to hear and repeat words spoken in } \\
\text { raised voice at } 1 \text { meter }\end{array}$ & Hearing aids usually recommended \\
\hline 3 - Severe impairment & $61-80 \mathrm{~dB}$ (better ear) & $\begin{array}{l}\text { Able to hear some words when shouted } \\
\text { into better ear }\end{array}$ & $\begin{array}{l}\text { Hearing aids needed, If no hearing aids } \\
\text { available, lip reading and signing should be } \\
\text { taught }\end{array}$ \\
\hline $\begin{array}{l}4 \text { - Profound impairment } \\
\text { including deafness }\end{array}$ & $81 \mathrm{~dB}$ ore greater (better ear) & Unable to hear even at shouted voice & $\begin{array}{l}\text { Hearing aids may help understanding words. } \\
\text { Additional rehabilitation needed. Lip reading } \\
\text { and sometimes signing essential }\end{array}$ \\
\hline
\end{tabular}

Grades 2, 3 and 4 are classified as disabling hearing imapirment. The audiometric ISO values are averages of values at 500, 1000, 2000 and $4000 \mathrm{~Hz}$.

Source: World Health Organization. (2014). Grades of hearing impairment. Retrieved October 10, 2014, from http://www.who.int/pbd/deafness/hearing_impairment_grades/en/index.html 